\title{
Expression and localisation of methylthioadenosine phosphorylase (MTAP) in oral squamous cell carcinoma and their significance in epithelial-to-mesenchymal transition
}

\author{
Yusuke Amano $^{1}$, Daisuke Matsubara ${ }^{1}$, Atsushi Kihara ${ }^{1}$, Hiroshi Nishino ${ }^{2}$, \\ YOSHIYUKI MORI ${ }^{3}$, TOSHIRO NIKI ${ }^{1}$ \\ ${ }^{1}$ Department of Integrative Pathology, Jichi Medical University, Shimotsuke, Japan; \\ ${ }^{2}$ Department of Otolaryngology, Jichi Medical University, Shimotsuke, Japan; ${ }^{3}$ Department of \\ Dentistry, Oral and Maxillofacial Surgery, Jichi Medical University, Shimotsuke, Japan
}

\begin{abstract}
Summary
Methylthioadenosine phosphorylase (MTAP) is a ratelimiting enzyme in the methionine salvage pathway, which recycles one carbon unit that is lost during polyamine synthesis back into the methionine cycle. Although MTAP deficiency has been reported in various tumours, MTAP is overexpressed and might promote oncogenesis in other cancers, including prostate and colon cancer. Currently, little is known about the MTAP status of oral squamous cell carcinoma (OSCC). In this study, we immunohistochemically examined the expression of MTAP in surgically resected oral epithelial dysplasia (OED, $n=7$ ), carcinoma in situ (CIS) $(n=16)$, and OSCC $(n=118)$. In the normal epithelium, MTAP was only weakly expressed in the cytoplasm of the basal layer cells. In OED, CIS, and OSCC, MTAP was uniformly expressed in the cytoplasm of the dysplastic and cancer cells. In addition to cytoplasmic MTAP expression, 45 of 118 cases $(38.1 \%)$ exhibited increased nuclear expression of MTAP in the cancer cells at the invasive front. Statistical analysis showed that the concomitant nuclear and cytoplasmic expression of MTAP was associated with a high budding score $(p=0.0023)$; poor differentiation ( $p=0.0044)$; aggressive invasion patterns $(p=0.0001)$; and features of epithelial-to-mesenchymal transition (EMT), such as loss of E-cadherin expression ( $p=0.0003)$ and upregulated expression of vimentin $(p=0.0002)$, slug $(p=0.0002)$, and laminin 5 $(p<0.0001)$. High expression of protein arginine methyltransferase 1 or 5 , the functions of which are reported to be inhibited in MTAP-deficient cancer, was associated with the concomitant nuclear and cytoplasmic expression of MTAP $(p<0.0001)$. Concomitant nuclear and cytoplasmic expression of MTAP was marginally significantly associated with worse 5 -year relapse-free survival $(p=0.045)$. These findings suggest that MTAP not only plays a role in the oncogenesis of OSCC, but that it might also make it more aggressive by inducing EMT through its activity in the methionine salvage pathway.
\end{abstract}

Key words: MTAP; oral squamous cell carcinoma; epithelial-to-mesenchymal transition.

Received 30 July 2020, revised 22 May, accepted 30 May 2021 Available online 10 September 2021

\section{INTRODUCTION}

Oral squamous cell carcinoma (OSCC) is one of the most common types of malignant head and neck cancer. ${ }^{1}$ Usually, patients with head and neck squamous cell carcinoma (HNSCC), including OSCC, already have advanced disease at the time of their initial diagnosis, with regional lymph node metastasis and distant metastasis seen in $43 \%$ and $10 \%$ of patients, respectively. ${ }^{2}$ Despite the advent of chemotherapy, radiotherapy, and multimodal therapy, the 5-year overall survival (OS) rate of HNSCC has remained approximately $50 \%$ for the past 30 years. ${ }^{3}$ No specific diagnostic markers of OSCC have yet been identified.

The enzyme methylthioadenosine phosphorylase (MTAP) cleaves methylthioadenosine (MTA), which is generated during the biosynthesis of polyamines, to produce adenine and 5-methylthioribose-1-phosphate. The 5-methylthioribose-1-phosphate is further metabolised to methionine, while the adenine is converted to AMP. ${ }^{4}$ This methionine salvage pathway plays an important role in maintaining methionine and nucleotide pools, which are both essential for rapidly growing cells and metabolically stressed cells. ${ }^{5}$

The MTAP gene, which is located at chromosomal locus $9 \mathrm{p} 21$, is frequently deleted in human cancers due to its proximity to the tumour suppressor gene cyclin-dependent kinase inhibitor 2A (CDKN2A). ${ }^{4}$ MTAP deficiency occurs frequently in haematological malignancies and various solid tumours, including malignant melanoma, hepatocellular carcinoma, malignant mesothelioma, non-small cell lung cancer, renal cell carcinoma, breast cancer, and glioma, suggesting that MTAP might have a tumour-suppressing role in these types of cancer. ${ }^{4}$ However, MTAP is frequently overexpressed in other cancers, such as prostate and colon cancer, in which it might promote oncogenesis. ${ }^{6-9}$

From a therapeutic viewpoint, cells lacking MTAP are unable to salvage adenine or methionine from MTA, ${ }^{5,10}$ and this might lead to therapeutic vulnerability in MTAPdeficient tumours. In fact, recent studies have shown that in cells lacking MTAP, the accumulation of its substrate MTA inhibited the expression of protein arginine methyltransferase (PRMT) 1 and 5, resulting in increased sensitivity to PRMT5 depletion. $^{11-13}$ 
Table 1 Antibodies used for immunohistochemical examination

\begin{tabular}{|c|c|c|c|c|c|}
\hline Primary antibody & Clone or code & Type & Source & Dilution & Pretreatment \\
\hline MTAP & clone EPR6893 & Rabbit monoclonal & Abcam & $1 / 100$ & Autoclaved in $10 \mathrm{mM}$ citrate buffer $(\mathrm{pH} 9.0)$ at $120^{\circ} \mathrm{C}$ for $10 \mathrm{~min}$ \\
\hline PRMT5 & HPA005525 & Rabbit polyclonal & Sigma-Aldrich & $1 / 100$ & Autoclaved in $10 \mathrm{mM}$ citrate buffer $(\mathrm{pH} 9.0)$ at $120^{\circ} \mathrm{C}$ for $10 \mathrm{~min}$ \\
\hline p16 & Clone E6H4 & Mouse monoclonal & Roche & Prediluted & CC1-standard (Ventana Discovery XT automated stainer) \\
\hline E-cadherin & Clone 36 & Mouse monoclonal & BD Biosciences & $1 / 400$ & $10 \mathrm{mM}$ citrate buffer (pH6.0) at $95^{\circ} \mathrm{C}$ for $10 \mathrm{~min}$ \\
\hline Vimentin & Clone V9 & Mouse monoclonal & DakoCytomation & $1 / 400$ & $\begin{array}{l}\text { Incubated in a microwave in } 10 \mathrm{mM} \text { citrate buffer }(\mathrm{pH} 6.0) \text { at } 95^{\circ} \mathrm{C} \\
\text { for } 10 \mathrm{~min}\end{array}$ \\
\hline Slug & Clone C19G7 & Rabbit monoclonal & Cell signaling & $1 / 50$ & Autoclaved in $10 \mathrm{mM}$ citrate buffer $(\mathrm{pH} 7.0)$ at $120^{\circ} \mathrm{C}$ for $10 \mathrm{~min}$ \\
\hline Laminin 5 & Clone 1-97 & Mouse monoclonal & $\begin{array}{l}\text { Described in a } \\
\text { previous } \\
\text { study }^{35}\end{array}$ & $1 / 50$ & Autoclaved in $10 \mathrm{mM}$ citrate buffer $(\mathrm{pH} 9.0)$ at $120^{\circ} \mathrm{C}$ for $10 \mathrm{~min}$ \\
\hline
\end{tabular}

To date, although MTAP deficiency has been reported in nasopharyngeal SCC, the MTAP status of OSCC has not been elucidated. ${ }^{14}$ In this study, we immunohistochemically examined the expression and localisation of MTAP in OSCC, carcinoma in situ (CIS), and its precursor lesion, oral epithelial dysplasia (OED), and sought to clarify the clinicopathological significance of MTAP expression in OSCC.

\section{MATERIALS AND METHODS}

The study protocol was approved by the ethics committee of Jichi Medical University (approval ID: A15-269).

See Supplementary Data for supporting information on Materials and Methods.

\section{Patients and tumours}

Tumour specimens were obtained from 141 patients who underwent head and neck cancer surgery without neoadjuvant chemoradiotherapy at Jichi Medical University Hospital, Japan, between 2010 and 2015. The patients were suffering from OED (corresponding to moderate dysplasia) ( $n=7)$, CIS $(n=16)$, or OSCC $(n=118)$. All histological diagnoses were made according to the World Health Organization (WHO) 2005 criteria. ${ }^{15}$ Information regarding the following factors was collected for each case: age, sex, primary site, histological grade, tumour stage, lymph node metastasis, Yamamoto-Kohama (YK) classification, ${ }^{16}$ lymphovascular invasion, perineural invasion, and patient survival. The patient cohort consisted of 73 males and 68 females, ranging in age between 26 and 89 years (mean age 64.4 years). The primary sites were as follows: tongue $(n=101)$, gingiva $(n=22)$, buccal mucosa $(n=6)$, oral floor $(n=11)$, and palate $(n=1)$. The pathological stage was defined according to the TNM classification established by the American Joint Committee on Cancer (AJCC) and the International Union Against Cancer (UICC), ${ }^{17}$ and the cut-off values for depth of invasion (DOI) and tumour thickness were also based on this classification. ${ }^{17}$ The follow-up period ranged in duration from 81 to 3441 days (median 1520 days).

The morphological pattern of invasion was determined according to the YK classification as follows: grade 1, a well-defined borderline; grade 2, a less prominent borderline; grade 3 , a group of cells and no distinct borderline; grade $4 \mathrm{C}$, diffuse invasion of the cord-like type; grade 4D, diffuse invasion of the widespread type. ${ }^{16}$

Tumour budding was defined as the presence of isolated neoplastic cells or small clusters of cells with $<5$ cells at the invasive front. ${ }^{18}$ Initially, the specimens were assessed using the lowest power objective lens to identify the areas with the highest tumour-budding intensity. Subsequently, using a $\times 20$ objective lens, the number of tumour buds (the number of isolated neoplastic cells or small clusters of $<5$ cells) was counted in just one $\times 200$ power field of the chosen area. The intensity of tumour budding was then categorised as either high ( $\geq 5$ tumour buds detectable in one $\times 200$ power field), or low $(<5$ tumour buds detectable in one $\times 200$ power field). ${ }^{18}$

\section{Immunohistochemistry}

The specimens for the immunohistochemical evaluations were collected from representative areas of the tumours. Immunohistochemistry was performed using primary antibodies against the following antigens: MTAP, PRMT1, PRMT5, p16, E-cadherin, vimentin, slug, and laminin 5. Details of the antibodies used, antigen retrieval methods, and antibody dilutions are listed in Table 1.

The immunoperoxidase polymer method (Histofine Simple Stain MAX PO; Nichirei, Japan) was used to detect all primary antibodies, except the antibody against p16. For p16 immunostaining, the sections were stained with an automated immunostainer (Ventana BenchMark XT; Roche, Switzerland), according to the manufacturer's instructions. 3,3'-diaminobenzidine tetrahydrochloride (DAB) was used as a chromogen, and haematoxylin was used as a light counterstain.

\section{Evaluation of immunohistochemical findings}

The immunohistochemical staining was independently analysed by two pathologists (YA and DM), who were not aware of the patients' clinical data, through microscopic examinations. MTAP immunoreactivity was evaluated using a previously described method. ${ }^{19}$ Inflammatory cells, including macrophages and lymphocytes, fibroblasts, and endothelial cells, served as internal positive controls for MTAP in each staining protocol. ${ }^{20}$ Vimentin, PRMT1, PRMT5, E-cadherin, and slug immunoreactivity were evaluated using previously described methods. ${ }^{18,21-25}$ p16 immunoreactivity was considered positive if $>70 \%$ of the tumour cells displayed moderate or strong and diffuse nuclear staining. ${ }^{26}$

Laminin 5 staining was semi-quantitatively divided into four categories: 0 , no immunostaining; $1+,<10 \%$ reactive cells; $2+, 11-50 \%$ reactive cells; and $3+,>50 \%$ reactive cells. For the statistical analysis, it was further categorised as low $(0$ and $1+)$ or high $(2+$ and $3+)$, based on the method reported by Marangon et al. ${ }^{27}$

\section{Statistical analysis}

The chi-squared test or Fisher's exact test was used to evaluate the associations among MTAP localisation, clinicopathological factors, epithelial-tomesenchymal transition (EMT) marker expression, and PRMT expression. Disease-free survival and OS rates were calculated according to the Kaplan-Meier method, and differences between groups were assessed using the log-rank test. Univariate and multivariate analyses were carried out using the Cox proportional hazards model [described as hazard ratios with $95 \%$ confidence intervals $(95 \% \mathrm{CI})$ together with $p$ values]. $p$ values of $<0.05$ were regarded as statistically significant. All statistical analyses were performed using the statistical software BellCurve for Excel (Social Survey Research Information Co Ltd, Japan).

\section{RESULTS}

\section{Expression of MTAP in the normal squamous epithelium, OED, and CIS}

First, we examined the MTAP staining patterns in the normal epithelium (the uninvolved area of the tongue in the OED case), OED, and CIS. The cytoplasm of the basal cells of the normal squamous epithelium was slightly positive for MTAP (Fig. 1A). In six OED cases, uniformly positive MTAP 
staining was detected in the cytoplasm of the dysplastic cells. In the remaining case of OED, both the cytoplasm and nuclei of the dysplastic cells were MTAP-positive (Table 2). The MTAP staining intensity of the OED tissue was nearly equal to that of the normal epithelium (Fig. 1B). In 15 CIS cases, uniformly positive MTAP staining was detected in the cytoplasm of the neoplastic cells (Fig. 1C, Table 2). In the remaining case of CIS, both the cytoplasm and nuclei of the neoplastic cells were positively stained for MTAP. The MTAP staining intensity did not differ significantly among the normal epithelium, OED, and CIS.

\section{Expression and localisation of MTAP in invasive OSCC}

In invasive OSCC, essentially all cases exhibited moderate to strong MTAP expression in the cytoplasm of the cancer cells. However, in a subset of invasive OSCC cases, we noted a tendency for both nuclear and cytoplasmic MTAP expression to be seen in the cancer cells at the invasive front. Representative cases are shown in Fig. 2A. In the cases with welldefined invasive fronts (YK-2), the cancer cell cytoplasm was uniformly positive for MTAP (Fig. 2A). In the cases involving diffuse invasion of the cord-like type (YK-4C) or diffuse invasion of the widespread type (YK-4D), the cancer cells often displayed diffuse positivity for MTAP in both the nucleus and cytoplasm (Fig. 2B,C). The staining intensity was stronger in the $\mathrm{YK}-4 \mathrm{C}$ and $\mathrm{YK}-4 \mathrm{D}$ cases than in the $\mathrm{YK}$ 2 cases. These results suggested that the concomitant nuclear and cytoplasmic localisation of MTAP is related to invasiveness in OSCC.

As virtually all of the invasive OSCC cases were positively stained for MTAP, we decided to classify the cases into two groups according to their MTAP localisation patterns, i.e., into the cytoplasmic group and the cytoplasmic and nuclear group. We then examined the significance of the concomitant cytoplasmic and nuclear localisation of MTAP.

The associations between MTAP localisation and clinicopathological factors in OSCC are shown in Table 2. The concomitant nuclear and cytoplasmic localisation of MTAP was not associated with sex, tumour location, the DOI, lymphovascular invasion, neural invasion, or lymph node metastasis (Table 2). Tumour locations in the tongue, i.e., the oral tongue or the base of the tongue, were not associated with MTAP localisation (Supplementary Table 1, Appendix A). As described above, OED and CIS were clearly associated with cytoplasmic MTAP expression $(p=0.0061)$. The concomitant localisation of MTAP in the nucleus and cytoplasm was marginally significantly associated with an advanced pT stage (pT4 vs pT1-3) and tumour size (tumour size $>20 \mathrm{~mm}$ vs $\leq 20 \mathrm{~mm}) \quad(p=0.0225$ and 0.0421 , respectively).

We then focused on the morphological pattern of the invasive front, according to the YK classification, and examined its relationship with MTAP localisation. Among the YK-1, YK-2, and YK-3 cases, MTAP was localised in the cytoplasm in most cases (46 of 58 cases), with 12 cases showing concomitant nuclear and cytoplasmic localisation (Table 2). Among the YK-4C and YK-4D cases, MTAP was localised in the cytoplasm alone in 27 of 60 cases and in both the nucleus and cytoplasm in 33 of 60 cases (Table 2). Thus, the concomitant localisation of MTAP in the nucleus and cytoplasm was associated with the YK-4C and YK-4D invasion patterns $(p=0.0001)$ and also with tumour budding $(p=0.0023)$. The frequencies of nuclear MTAP localisation in the low- and high-budding cases were $33.3 \%$ (35/105 cases) and $76.9 \%$ (10/13 cases), respectively. The concomitant localisation of MTAP in the nucleus and cytoplasm was also associated with a higher histological grade $(p=0.0044)$, i.e., it was seen in 8 of 10 grade 3 cases and 37 of 108 grade 1/2 cases.

\section{Associations between MTAP localisation and the expression of EMT markers and PRMT1 and PRMT5}

Our results suggested that nuclear localisation of MTAP is associated with aggressiveness of OSCC. In OSCC, the mode of invasion at the invasive front is a histological predictor of recurrence and prognosis. ${ }^{16}$ An infiltrative growth pattern, as exemplified by the presence of isolated cancer cells and/or small nests of cancer cells at the stromal interface, was found to be associated with the loss of the epithelial marker Ecadherin and the upregulation of vimentin, slug, and laminin 5 expression and was considered to be indicative of EMT. ${ }^{28}$ We previously showed that the expression of PRMT5, a type II arginine methyltransferase, was associated with EMT in OSCC. ${ }^{23}$ PRMT1, another arginine methyltransferase, has also been reported to be associated with EMT. ${ }^{29}$

Thus, we stained adjacent sections for E-cadherin, vimentin, slug, laminin 5, PRMT1, and PRMT5 and examined whether the expression levels of these molecules and/or the localisation of MTAP were associated with the cancer cell invasion pattern.
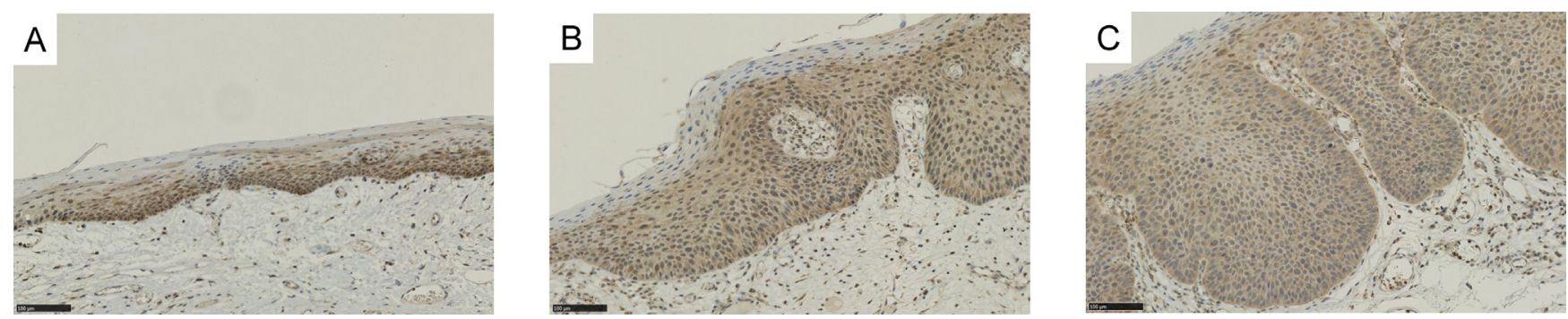

Fig. 1 Expression of MTAP in the normal epithelium, oral epithelial dysplasia (OED), and carcinoma in situ (CIS). (A) Cytoplasmic expression of MTAP in the basal and parabasal cell layer of the normal epithelium. (B) Cytoplasmic expression of MTAP in dysplastic cells. (C) Cytoplasmic expression of MTAP in neoplastic cells. Bar $=100 \mu \mathrm{m}$. 
Table 2 Correlation between MTAP localisation and clinicopathological factors

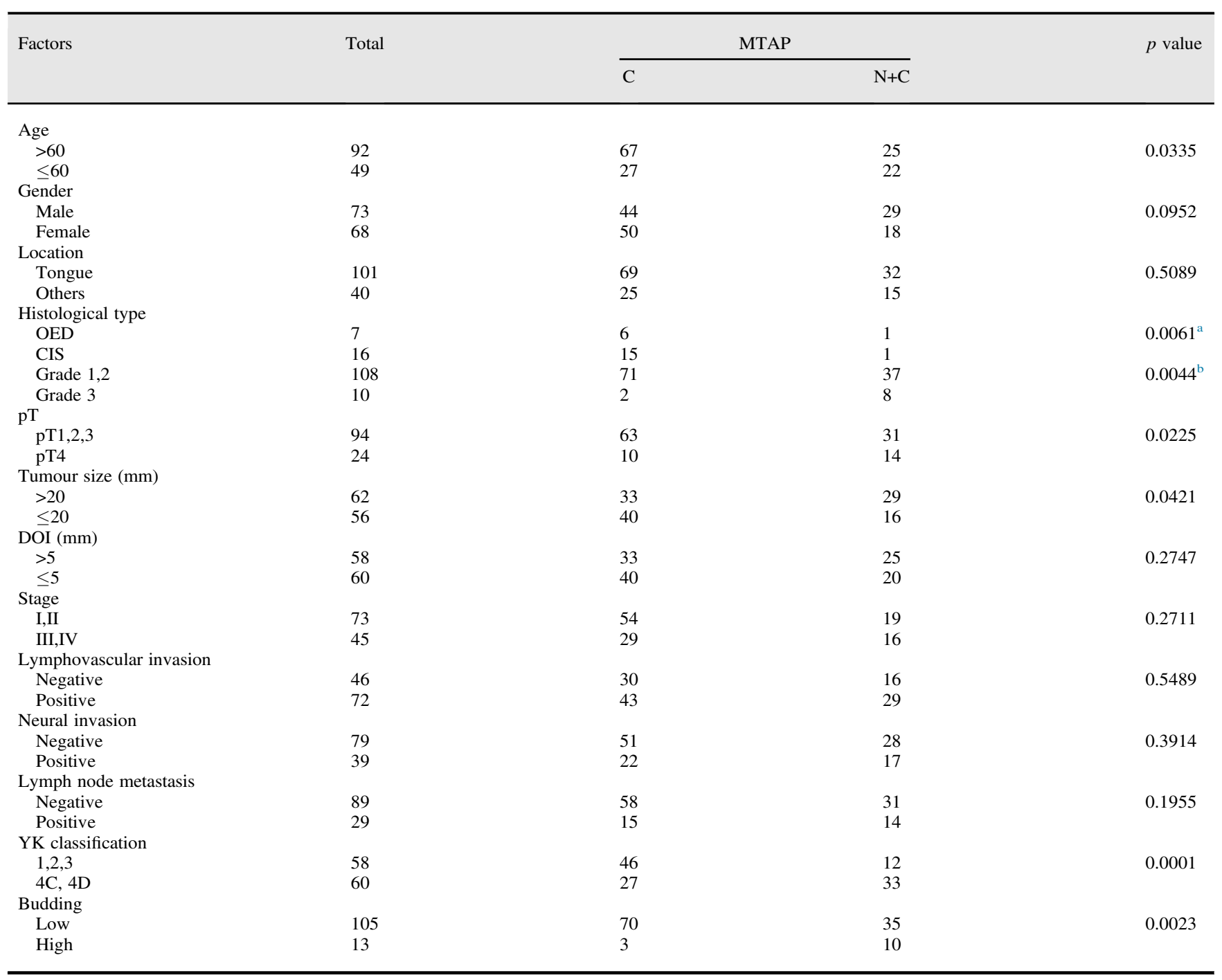

Age, gender and location including OED and CIS.

CIS, carcinoma in situ; DOI, depth of invasion; OED, oral epithelial dysplasia; YK, Yamamoto-Kohama.

${ }^{a}$ OED, CIS vs Grade $1-3$.

b Grade 1,2 vs Grade 3.
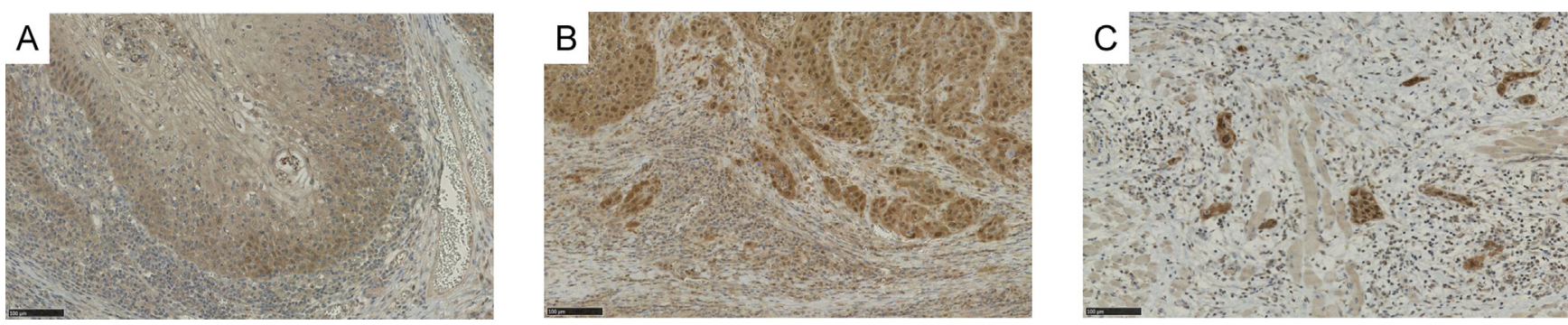

Fig. 2 Expression of MTAP in SCC in the invasive front. (A) Cytoplasmic expression of MTAP in YK-2. (B) Strong cytoplasmic and nuclear expression of MTAP in YK-4C. (C) Strong cytoplasmic and nuclear expression of MTAP in YK-4D. Bar=100 $\mu \mathrm{m}$.

In the YK-3 cases, the cytoplasm of the cancer cells was MTAP-positive (Fig. 3A), and the cancer cell membranes were E-cadherin-positive (Fig. 3B). On the other hand, the cancer cells were negative for vimentin (Fig. 3C), slug (Fig. 3D), and laminin 5 (Fig. 3E). PRMT1 was weakly expressed in the nuclei and cytoplasm of the cancer cells
(Fig. 3F), while PRMT5 was expressed in the cytoplasm of the cancer cells (Fig. 3G).

In the YK-4C cases, both the nuclei and cytoplasm of the cancer cells were MTAP-positive (Fig. 3H). E-cadherin expression was decreased in the membranes of the cancer cells (Fig. 3I), while the cancer cells were vimentin-positive 
(Fig. 3J). Slug was expressed in the nuclei of the cancer cells (Fig. 3K). Laminin 5 was diffusely expressed in the cytoplasm of the cancer cells (Fig. 3L). Both PRMT1 and PRMT5 were highly expressed in the nuclei and cytoplasm of the cancer cells (Fig. 3M,N, respectively).

Statistical analyses showed that reduced expression of Ecadherin and high expression of vimentin, slug, laminin 5, PRMT1, and PRMT5 were significantly associated with the concomitant localisation of MTAP in both the nucleus and cytoplasm (Table 3). p16 expression was not correlated with MTAP expression (Table 3).

\section{Association between MTAP localisation and OSCC prognosis}

During the follow-up period, 46 of 117 patients suffered local or metastatic recurrence. The 5-year relapse-free survival (RFS) rate was $61.9 \%$ in the cytoplasmic MTAP cases and $46.6 \%$ in the nuclear and cytoplasmic MTAP cases. The concomitant expression of MTAP in both the nucleus and cytoplasm was associated with RFS (with marginal statistical significance; $p=0.045$ ) (Fig. 4A). The 5-year OS rate was $83.3 \%$ in the cytoplasmic MTAP cases, and $77.7 \%$ in the nuclear and cytoplasmic MTAP cases. MTAP localisation was not significantly associated with OS $(p=0.45)$ (Fig. 4B).

Next, we performed univariate and multivariate Cox proportional hazards analyses to identify parameters that affect RFS in OSCC patients. Univariate analysis revealed that histological type $(p=0.0023)$, tumour size $(p=0.0167)$, DOI $(p=0.0003)$, disease stage $(p=0.0278)$, lymphovascular invasion $(p=0.0038)$, neural invasion $(p=0.0001)$, YK classification $(p=0.0017)$, budding grade $(p=0.0006)$, and MTAP localisation $(\mathrm{p}=0.0485)$ were significantly associated with RFS in OSCC patients. However, in the multivariate analysis only neural invasion continued to exhibit a statistically significant association with RFS ( $p=0.0109$ ) (Table 4).

\section{DISCUSSION}

In the present study, we immunohistochemically analysed MTAP expression in OED, CIS, and OSCC. As a result, we found that MTAP was consistently expressed in the cytoplasm of the basal cells of the normal epithelium, the dysplastic cells of OED, and cancer cells. These findings suggest that MTAP not only plays a physiological function in basal cells, but is also involved in the oncogenesis of OSCC from an early stage. In addition to cytoplasmic MTAP expression, both nuclear and cytoplasmic MTAP expression were observed in some cases, particularly in cases involving an advanced pT stage (pT4), a larger tumour size (>20 mm), or an infiltrative invasion pattern (YK-4C or YK-4D). The concomitant expression of MTAP in both the nucleus and cytoplasm was associated with relapse. Collectively, these results suggest that MTAP might be involved in oncogenesis and aggressiveness in OSCC.

MTAP is a rate-limiting enzyme in the methionine salvage pathway, which is critical for maintaining adenosine and methionine pools. ${ }^{5}$ Methionine is involved in many essential cell functions, including protein synthesis, methylation reactions, redox maintenance, and polyamine biosynthesis, and the methionine cycle is also coupled to the folate cycle, producing further links between methionine and purine/pyrimidine synthesis. ${ }^{5}$ The diverse roles of methionine indicate that the methionine salvage pathway, and hence MTAP, might play important roles in rapidly proliferating and metabolically stressed cells, especially cancer cells. ${ }^{5}$ Indeed, recent studies have shown that MTAP expression is upregulated in prostate cancer, promoting its in vitro and in vivo growth. ${ }^{6,7}$ Pharmacological inhibition of MTAP reduced the growth of prostate cancer cells, indicating that MTAP might be a therapeutic target for treatments for prostate cancer. ${ }^{6,7}$ In colon cancer, MTAP expression is upregulated by LEF/TCF/beta-catenin, in parallel with tumour progression and EMT. ${ }^{8,9}$ EMT is a complex and reversible biological process, by which an epithelial tumour alters its polar, adhesive phenotype to a mesenchymal phenotype characterised by increases in cell migration and invasive potential, cytoskeletal remodelling, and resistance to apoptosis. ${ }^{28}$ In our study, the cases of OSCC in which the cancer cells exhibited MTAP expression in both their nuclei and cytoplasm were associated with an infiltrative invasion pattern, the loss of epithelial markers (E-cadherin), and the upregulation of mesenchymal marker expression (vimentin, slug, and laminin 5). These results are in line with the findings obtained for colon and prostate cancer and suggest that MTAP might be a therapeutic target for treatments for OSCC as well.

The MTAP gene is frequently co-deleted with the tumour suppressor gene CDKN2A in various human cancers. ${ }^{4}$ Given the important role of the methionine salvage pathway, MTAP deficiency might lead to metabolic vulnerabilities that could be exploited during the development of cancer therapies. ${ }^{5}$ Indeed, recent studies have shown that MTA, the substrate of MTAP, accumulates in MTAPdeficient cells, resulting in the inhibition of the expression of members of the PRMT family, including type I (e.g., PRMT1) and type II (e.g., PRMT5) PRMTs. ${ }^{11-13}$ Hence, PRMT5 depletion by RNA interference had lethal effects on MTAP-deficient cells. ${ }^{11-13}$
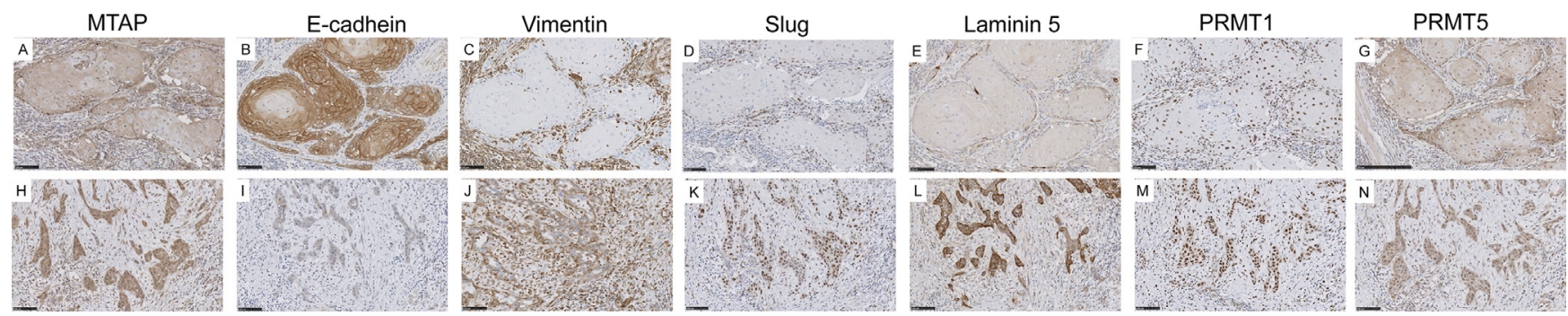

Fig. 3 Expression of MTAP, E-cadherin, vimentin, slug, laminin 5, PRMT1, and PRMT5 in the invasive front of SCC. (A) Cytoplasmic expression of MTAP in YK-3 (B) Strong membranous expression of E-cadherin in YK-3, (C) Cytoplasmic expression in stromal cells of vimentin in YK-3. (D) Negative expression of slug in YK-3. (E) Negative expression of laminin 5 in YK-3. (F) Low nuclear expression of PRMT1 in YK-3. (G) Cytoplasmic expression of PRMT5 in YK-3. (H) Strong nuclear and cytoplasmic expression of MTAP in YK-4C. (I) Decreased membranous expression of E-cadherin in YK-4C. (J) Cytoplasmic expression in both cancer cells and stromal cells of vimentin in YK-4C. (K) Nuclear expression of slug in YK-4C. (L) Cytoplasmic expression of laminin 5 in YK-4C. (M) High nuclear expression of PRMT1 in YK-4C. (N) Nuclear and cytoplasmic expression of PRMT5 in YK-4C. (A-F,H-N) Bar=100 $\mu \mathrm{m}$; (G) Bar=250 $\mu \mathrm{m}$. 
Table 3 Correlation among MTAP localisation, EMT markers, and PRMT expression

\begin{tabular}{|c|c|c|c|c|}
\hline & & \multicolumn{2}{|c|}{ MTAP } & \multirow[t]{2}{*}{$p$ value } \\
\hline & & $\mathrm{C}$ & $\mathrm{N}+\mathrm{C}$ & \\
\hline \multirow[t]{2}{*}{ E-cadherin } & Low & 24 & 30 & 0.0003 \\
\hline & High & 49 & 15 & \\
\hline \multirow[t]{2}{*}{ Vimentin } & Negative & 44 & 11 & 0.0002 \\
\hline & Positive & 29 & 34 & \\
\hline \multirow[t]{2}{*}{ Slug } & Low & 34 & 6 & 0.0002 \\
\hline & High & 39 & 39 & \\
\hline \multirow[t]{2}{*}{ Laminin 5} & Low & 49 & 11 & $<0.0001$ \\
\hline & High & 24 & 34 & \\
\hline \multirow[t]{2}{*}{ PRMT1 } & Low & 44 & 9 & $<0.0001$ \\
\hline & High & 29 & 36 & \\
\hline \multirow[t]{2}{*}{ PRMT5 } & $\mathrm{C}$ & 61 & 20 & $<0.0001$ \\
\hline & $\mathrm{N}+\mathrm{C}$ & 12 & 25 & \\
\hline \multirow[t]{2}{*}{ p16 } & Negative & 67 & 40 & 0.5997 \\
\hline & Positive & 6 & 5 & \\
\hline
\end{tabular}

C, cytoplasm; N, nuclear.
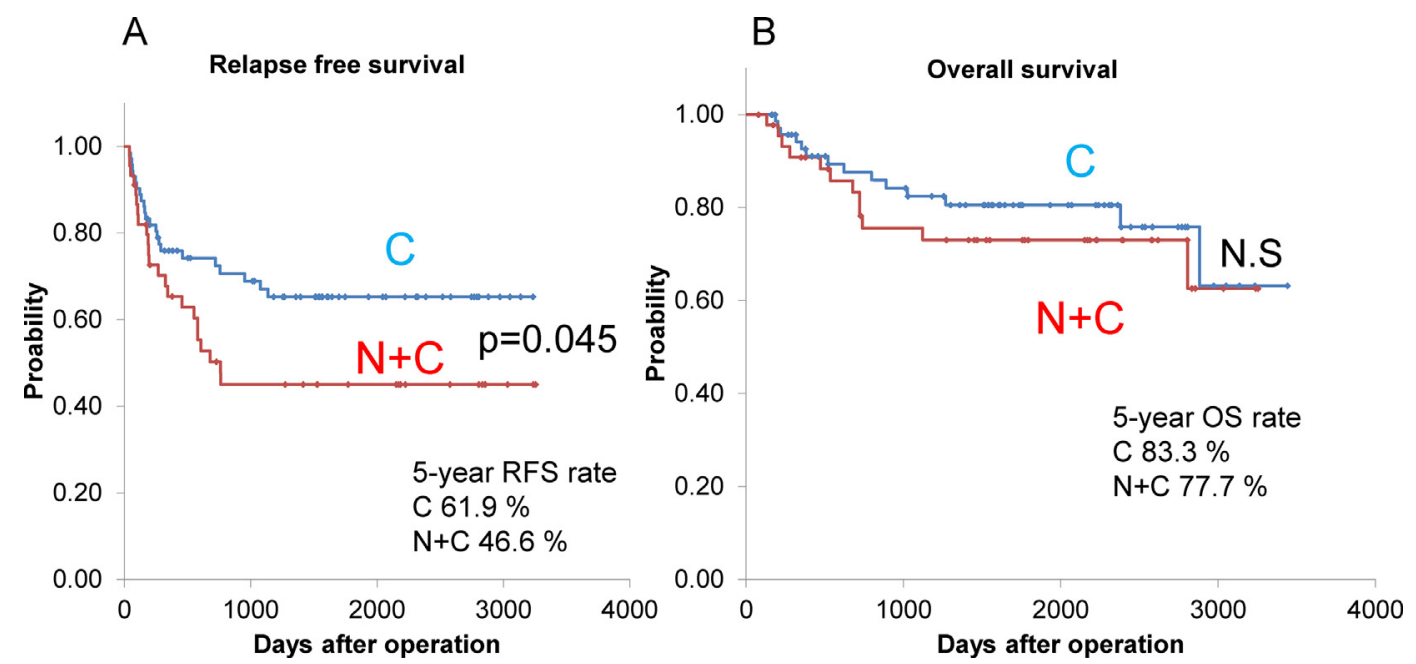

Fig. 4 (A) Kaplan-Meier curves for relapse-free survival of patients with MTAP expression. (B) Kaplan-Meier curves for overall survival of patients with MTAP expression.

PRMTs transfer methyl groups to the guanidine nitrogen molecules of the arginine residues on their target proteins, and this process alters gene expression, signal transduction, RNA splicing, and other cellular functions. ${ }^{30,31} \mathrm{We}$ previously reported that the nuclear localisation of PRMT5 is involved in EMT in OSCC. ${ }^{23}$ In gastric and breast cancer, PRMT1 was also found to be a regulator of EMT. ${ }^{29,32,33}$ These findings raise the interesting possibility that MTAP and PRMTs might be regulated in a coordinated manner in aggressive cancer cells that undergo EMT.

Our study was limited by the fact that our results were largely correlative. Thus, the precise molecular mechanisms by which MTAP and PRMTs contribute to EMT remain unknown. Also, no genetic analysis of deletions at the CDKN2A/MTAP locus was performed in this study cohort. We sought to compensate for this limitation by referring to the publicly available database of HNSCC (The Cancer Genome Atlas, TCGA) ${ }^{34}$ via c-BioPortal (www.cbioportal.org). Analysis of the TCGA cohort revealed that the genetic deletion of CDKN2A and MTAP occurred in $26.0 \%$ and $13.6 \%$ of HNSCC, respectively (Supplementary Fig. 1A,B, Supplementary Table 2, Appendix A). Co-deletion of CDKN2A and MTAP occurred in $12.5 \%$. Thus, about half of CDKN2A deleted cases retained the MTAP locus. Gene expression data also showed that the large majority of HNSCC expressed MTAP mRNA (Supplementary Fig. 1C, Appendix A). These results are in accordance with our immunohistochemical data showing expression of MTAP in most OSCC cases.

Other potential critique would be about the specificity of the MTAP antibody used in this study. Also, nuclear localisation of MTAP may need to be confirmed by other method. To address these issues, we additionally performed western blot and immunofluorescent analysis using OSCC cell lines (Supplementary Fig. 2 and 3, Appendix A). Western blot analysis showed that administration led to the detection of an anti-MTAP antibody detected a single band corresponding to the MTAP protein in OSCC cell lines and HeLa cells, but this band disappeared in HeLa cells made 
Table 4 Univariate and multivariate analysis of relapse-free survival

\begin{tabular}{|c|c|c|c|c|c|c|}
\hline \multirow[t]{2}{*}{ Factors } & \multicolumn{2}{|c|}{ Univariate analysis } & \multirow[t]{2}{*}{$p$ value } & \multicolumn{2}{|c|}{ Multivariate analysis } & \multirow[t]{2}{*}{$p$ value } \\
\hline & HR & $95 \% \mathrm{CI}$ & & HR & $95 \% \mathrm{CI}$ & \\
\hline \multicolumn{7}{|l|}{ Age } \\
\hline$>60$ & 1 & & & & & \\
\hline$\leq 60$ & 1.08881 & $0.6048-1.9599$ & 0.7766 & & & \\
\hline \multicolumn{7}{|l|}{ Gender } \\
\hline Male & 0.86101 & $0.4818-1.5385$ & 0.6133 & & & \\
\hline \multicolumn{7}{|l|}{ Location } \\
\hline Tongue & 1 & & & & & \\
\hline Others & 1.3042 & $0.7035-2.4179$ & 0.3989 & & & \\
\hline \multicolumn{7}{|c|}{ Histological type } \\
\hline Grade 1,2 & 1 & & & 1 & & \\
\hline Grade 3 & 2.46185 & $1.3785-4.3964$ & 0.0023 & 1.13464 & $0.5657-2.2755$ & 0.722 \\
\hline \multicolumn{7}{|l|}{ pT } \\
\hline $1,2,3$ & 1 & & & & & \\
\hline \multicolumn{7}{|c|}{ Tumour size (mm) } \\
\hline$\leq 20$ & 1 & & & 1 & & \\
\hline$>20$ & 2.15655 & $1.1491-4.0469$ & 0.0167 & 1.42655 & $0.6787-2.9980$ & 0.3484 \\
\hline \multicolumn{7}{|l|}{ DOI (mm) } \\
\hline$\leq 5$ & 1 & & & 1 & & \\
\hline$>5$ & 3.23371 & $1.6984-6.1565$ & 0.0003 & 1.27059 & $0.5169-3.1227$ & 0.6016 \\
\hline \multicolumn{7}{|l|}{ Stage } \\
\hline I,II & 1 & & & 1 & & \\
\hline III,IV & 1.9465 & $1.0749-3.5243$ & 0.0278 & 0.73049 & $0.3423-1.5587$ & 0.4167 \\
\hline \multicolumn{7}{|c|}{ Lymphovascular invasion } \\
\hline Negative & 1 & & & 1 & & \\
\hline Positive & 2.81454 & $1.3955-5.6765$ & 0.0038 & 1.48494 & $0.6516-3.3835$ & 0.3467 \\
\hline \multicolumn{7}{|c|}{ Neural invasion } \\
\hline Negative & 1 & & & 1 & & \\
\hline Positive & 3.1419 & $1.7548-5.6253$ & 0.0001 & 2.38975 & $1.2218-4.6740$ & 0.0109 \\
\hline \multicolumn{7}{|c|}{ Lymph node metastasis } \\
\hline Negative & 1 & & & 1 & & \\
\hline Positive & 1.78971 & $0.9828-3.2590$ & 0.057 & 0.77671 & $0.3801-1.5867$ & 0.4881 \\
\hline \multicolumn{7}{|c|}{ YK classification } \\
\hline \multicolumn{7}{|l|}{ Budding } \\
\hline Low & 1 & & & 1 & & \\
\hline High & 3.29777 & $1.6643-6.534$ & 0.0006 & 1.93755 & $0.8431-4.4522$ & 0.1191 \\
\hline \multicolumn{7}{|l|}{ MTAP } \\
\hline $\mathrm{C}$ & 1 & & & 1 & & \\
\hline $\mathrm{N}+\mathrm{C}$ & 1.79017 & $1.0037-3.1929$ & 0.0485 & 1.14073 & $0.5683-2.2895$ & 0.711 \\
\hline
\end{tabular}

DOI, depth of invasion; HR, mean hazard ratio; YK, Yamamoto-Kohama; 95\% CI, 95\% confidence intervals.

deficient for MTAP by CRISPR/Cas9. Also, immunofluorescent analysis showed that while SAS cells without TGF$\beta$ treatment showed cytoplasmic MTAP expression, after TGF- $\beta$ treatment some SAS cells showed both nuclear and cytoplasmic MTAP expression. These additional data would support and reinforce our immunohistochemical data of MTAP expression in OSCC.

\section{CONCLUSIONS}

In conclusion, in OSCC, cytoplasmic MTAP expression is observed from an early stage of oncogenesis and persists throughout the progression of the disease, and the presence of both nuclear and cytoplasmic MTAP expression is associated with an aggressive invasion pattern and EMT. In OSCC cells, MTAP might promote tumour aggressiveness through its activity in the methionine salvage pathway.
Acknowledgements: We thank Tomoko Tamura and Sachiko Oguni for immunohistochemical and work and experiments. English language editing was provided by Medical English Service.

Conflicts of interest and sources of funding: This work was supported in part by JSPS KAKENHI Grant Number $19 \mathrm{~K} 10094$ (to YA). The authors state that there are no conflicts of interest to disclose.

\section{APPENDIX A. SUPPLEMENTARY DATA}

Supplementary data to this article can be found online at https://doi.org/10.1016/j.pathol.2021.05.101.

Address for correspondence: Dr Y. Amano, Department of Pathology, Jichi Medical University, 3311-1, Yakushiji, Shimotsuke, Tochigi, Japan. E-mail: amano.yusuke@jici.ac.jp 


\section{References}

1. Shield KD, Ferlay J, Jemal A, et al. The global incidence of lip, oral cavity, and pharyngeal cancers by subsite in 2012. CA Cancer J Clin 2017; 67: 51-64.

2. Noguti J, De Moura CFG, De Jesus GPP, et al. Metastasis from oral cancer: an overview. Cancer Genomics Proteomics 2012; 9: $329-36$.

3. Sasahira T, Kirita T, Kuniyasu H. Update of molecular pathobiology in oral cancer: a review. Int J Clin Oncol 2014; 19: 431-6.

4. Bertino JR, Waud WR, Parker WB, Lubin M. Targeting tumors that lack methylthioadenosine phosphorylase (MTAP) activity: current strategies. Cancer Biol Ther 2011; 11: 627-32.

5. Sanderson SM, Gao X, Dai Z, Locasale JW. Methionine metabolism in health and cancer: a nexus of diet and precision medicine. Nat Rev Cancer 2019; 19: 625-37.

6. Bistulfi G, Affronti HC, Foster BA, et al. The essential role of methylthioadenosine phosphorylase in prostate cancer. Oncotarget 2016; 7 : 14380-93.

7. Affronti HC, Rowsam AM, Pellerite AJ, et al. Pharmacological polyamine catabolism upregulation with methionine salvage pathway inhibition as an effective prostate cancer therapy. Nat Commun 2020; 11: 52.

8. Bataille F, Rogler G, Modes K, et al. Strong expression of methylthioadenosine phosphorylase (MTAP) in human colon carcinoma cells is regulated by TCF1/[beta]-catenin. Lab Invest 2005; 85: 124-36.

9. Zhong Y, Lu K, Zhu S, et al. Characterization of methylthioadenosin phosphorylase (MTAP) expression in colorectal cancer. Artif Cells Nanomed Biotechnol 2018; 46: 2082-7.

10. Li Y, Wang Y, Wu P. 5'-methylthioadenosine and cancer: old molecules, new understanding. J Cancer 2019; 10: 927-36.

11. Mavrakis KJ, McDonald ER, Schlabach MR, et al. Disordered methionine metabolism in MTAP/CDKN2A-deleted cancers leads to dependence on PRMT5. Science 2016; 351: 1208-13.

12. Kryukov GV, Wilson FH, Ruth JR, et al. MTAP deletion confers enhanced dependency on the PRMT5 arginine methyltransferase in cancer cells. Science 2016; 351: 1214-8.

13. Marjon K, Cameron MJ, Quang $\mathrm{P}$, et al. MTAP deletions in cancer create vulnerability to targeting of the MAT2A/PRMT5/RIOK1 axis Cell Rep 2016; 15: 574-87.

14. He H-L, Lee Y-E, Shiue Y-L, et al. Characterization and prognostic significance of methylthioadenosine phosphorylase deficiency in nasopharyngeal carcinoma. Medicine (Baltimore) 2015; 94: e2271.

15. Barnes L, Eveson JW, Reichart P, Sidransky D, editors. World Health Organization Classification of Tumours: Pathology and Genetics, Head and Neck Tumours. Lyon: IARC Press, 2005; 177-9.

16. Yamamoto E, Kohama G, Sunakawa H, et al. Mode of invasion, bleomycin sensitivity, and clinical course in squamous cell carcinoma of the oral cavity. Cancer 1983; 51: 2175-80.

17. Brierley JD, Gospodarowicz MK, Wittekind C, editors. UICC TNM Classification of Malignant Tumours. 8th ed. New York: Wiley Blackwell, 2017.

18. Wang C, Huang H, Huang Z, et al. Tumor budding correlates with poor prognosis and epithelial-mesenchymal transition in tongue squamous cell carcinoma. J Oral Pathol Med 2011; 40: 545-51.

19. de Menezes WP, Silva VAO, Gomes INF, et al. Loss of $5^{\prime}$-methylthioadenosine phosphorylase (MTAP) is frequent in high-grade gliomas; nevertheless, it is not associated with higher tumor aggressiveness. Cells 2020; 9: 492.

20. Kinoshita Y, Hamasaki M, Yoshimura M, et al. A combination of MTAP and BAP1 immunohistochemistry is effective for distinguishing sarcomatoid mesothelioma from fibrous pleuritis. Lung Cancer 2018; 125: 198-204.

21. Altan B, Yokobori T, Ide M, et al. Nuclear PRMT1 expression is associated with poor prognosis and chemosensitivity in gastric cancer patients. Gastric Cancer 2016; 19: 789-97.

22. Ibrahim R, Matsubara D, Osman W, et al. Expression of PRMT5 in lung adenocarcinoma and its significance in epithelial-mesenchymal transition. Hum Pathol 2014; 45: 1397-405.

23. Amano Y, Matsubara D, Yoshimoto T, et al. Expression of protein arginine methyltransferase-5 in oral squamous cell carcinoma and its significance in epithelial-to-mesenchymal transition. Pathol Int 2018 68: 359-66.

24. Matsubara D, Kishaba Y, Yoshimoto T, et al. Immunohistochemical analysis of the expression of E-cadherin and ZEB1 in non-small cel lung cancer. Pathol Int 2014; 64: 560-8.

25. Kihara A, Wakana K, Kubota T, Kitagawa M. SLUG expression is an indicator of tumour recurrence in high-grade endometrial carcinomas. Histopathology 2016; 69: 374-82.

26. Vermorken JB, Psyrri A, Mesía R, et al. Impact of tumor HPV status on outcome in patients with recurrent and/or metastatic squamous cell carcinoma of the head and neck receiving chemotherapy with or without cetuximab: retrospective analysis of the phase III EXTREME trial. Ann Oncol 2014; 25: 801-7.

27. Marangon Jr H, Rocha VN, Leite CF, et al. Laminin-5 gamma 2 chain expression is associated with intensity of tumor budding and density of stromal myofibroblasts in oral squamous cell carcinoma. J Oral Pathol Med 2014; 43: 199-204.

28. Smith A, Teknos TN, Pan Q. Epithelial to mesenchymal transition in head and neck squamous cell carcinoma. Oral Oncol 2013; 49: $287-92$.

29. Katsuno Y, Qin J, Oses-Prieto J, et al. Arginine methylation of SMAD7 by PRMT1 in TGF- $\beta$-induced epithelial-mesenchymal transition and epithelial stem-cell generation. J Biol Chem 2018; 293: 13059-72.

30. Blanc RS, Richard S. Arginine methylation: the coming of age. Mol Cell 2017; 65: 8-24.

31. Stopa N, Krebs JE, Shechter D. The PRMT5 arginine methyltransferase: many roles in development, cancer and beyond. Cell Mol Life Sci 2015; 72: $2041-59$.

32. Zhang Y, Wang D, Zhang M, et al. Protein arginine methyltransferase 1 coordinates the epithelial-mesenchymal transition/proliferation dichotomy in gastric cancer cells. Exp Cell Res 2018; 362: 43-50.

33. Gao Y, Zhao Y, Zhang J, et al. The dual function of PRMT1 in modulating epithelial-mesenchymal transition and cellular senescence in breast cancer cells through regulation of ZEB1. Sci Rep 2016; 6: 19874.

34. Cancer Genome Atlas Network. Comprehensive genomic characterization of head and neck squamous cell carcinomas. Nature 2015; 517 $576-82$.

35. Niki T, Kohno T, Iba S, et al. Frequent co-localization of Cox-2 and laminin-5 gamma 2 chain at the invasive front of early-stage lung adenocarcinomas. Am J Pathol 2002; 160: 1129-41. 